\title{
Low-metallicity Star Formation: the characteristic mass and upper mass limit
}

\author{
Kazuyuki Omukai \\ National Astronomical Observatory of Japan, 2-21-1 Osawa, Mitaka, Tokyo 181-8588, Japan \\ email: omukai@th.nao.ac.jp
}

\begin{abstract}
We consider the fragmentation mass scale of low-metallicity clouds based on their thermal evolution. Then we present the protostellar evolution in the main accretion phase, and discuss the upper limit on the stellar mass by the stellar feedback.
\end{abstract}

Keywords. stars: formation, stars: Population II

\section{Characterictic Stellar Mass by Fragmentation}

The first stars in the universe were very massive, with a characteristic mass of $\gtrsim 100 M_{\odot}$. This statement has been confirmed by many numerical simulations (e.g., Bromm et al. 2002; Abel et al. 2002; Yoshida et al. 2008) and by analytical works (Omukai \& Palla 2002; McKee \& Tan 2008), and now has almost become a part of the standard theory. In the solar neighborhood, on the other hand, the initial mass function exhibits a peak around $0.1-1 M_{\odot}$. The origin of these characteristic masses can be attributed to the thermal evolution of pre-stellar clouds (e.g., Larson 2005).

In Figure 1, we show the temperature evolution of star-forming cores with different metallicities (Omukai et al. 2005). This is calculated by using a one-zone model assuming that the clouds collapse almost at a free-fall rate, the size of the cores is about the Jeans length, and the dust to metal ratio is the same as the local ISM value. The dotted lines indicate the constant Jeans mass.

The temperature of metal-free gas has a minimum around $10^{4} \mathrm{~cm}^{-3}$, where the rotational levels of $\mathrm{H}_{2}$ reach LTE and its cooling rate saturates. Up to this point, the rapid cooling allows the temperature to decrease with increasing density, i.e., the effective ratio of specific heat $\gamma=d \log p / d \log \rho<1$. In such a condition, clouds in shapes other than the sphere, e.g., filaments, which eventually fragment into smaller pieces, can also collapse gravitationally. However, once the temperature begins to increase with density, filamentary clouds no longer collapses due to increasing pressure support, and only spherical clouds can collapse thereafter. As a consequence, spherical clouds are formed at this moment. Since spherical clouds are hard to fragment, the typical epoch of fragmention is around this temperature minimum, and the Jeans mass there is imprinted as the characteristic fragmentation mass scale. In the case of zero-metallicity gas, this characteristic fragmentation mass is given by the Jeans mass around $10^{4} \mathrm{~cm}^{-3}$ and is about $1000 M_{\odot}$.

On the other hand, the solar-metallicity gas has a temperature minimum at $\sim 10^{5} \mathrm{~cm}^{-3}$. This is due to the cooling of the dust by infrared emission, which makes the temperature decrease until the thermal coupling of the gas and dust is reached. The Jeans mass at this temperature minimum is $\sim M_{\odot}$, which agrees with the observed characteristic mass in the solar neighborhood.

What are the processes in the slightly metal-enriched clouds? Below the metallicity $[\mathrm{M} / \mathrm{H}]=-6$, the temperature evolution is the same as that of zero-metallicity. With 


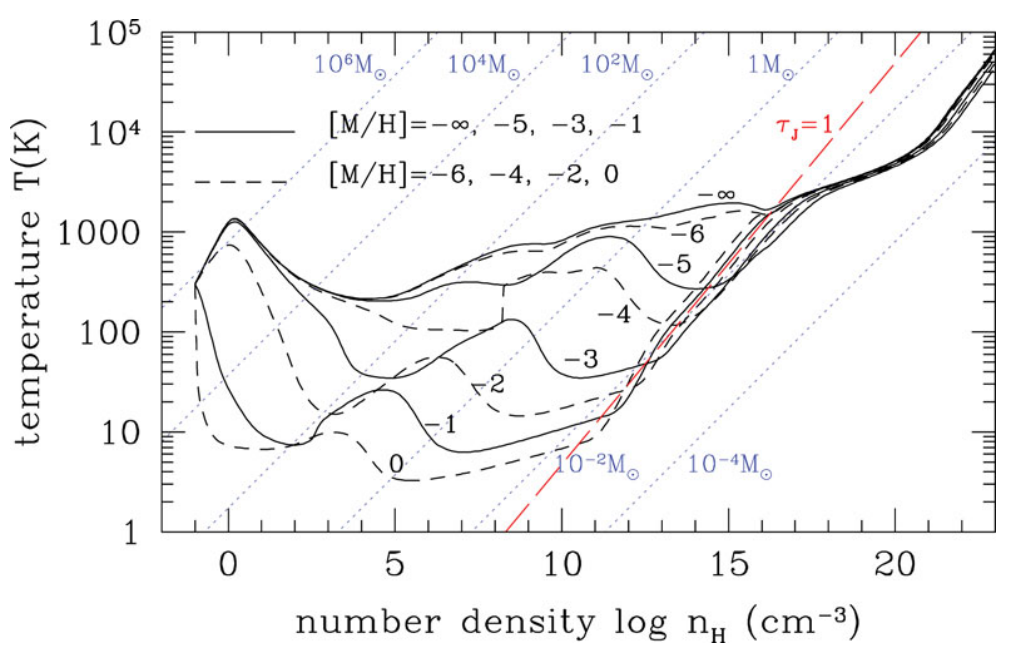

Figure 1. Temperature evolution of pre-stellar clouds with different metallicities. Those with metallicities $[\mathrm{M} / \mathrm{H}]=-\infty(\mathrm{Z}=0),-5,-3$, and $-1(-6,-4,-2$, and 0$)$ are shown by solid (dashed) lines. Only the present-day (3K) CMB is included as an external radiation field. The lines for constant Jeans mass are indicated by thin dotted lines. The positions where the central part of the clouds becomes optically thick to continuum self-absorption is indicated by the thin solid line. To the right of this line, the clouds are optically thick. (After Omukai et al. 2005)

metallicity of $[\mathrm{M} / \mathrm{H}]=-5$, a temperature dip appears in high density $\sim 10^{14} \mathrm{~cm}^{-3}$ owing to the dust cooling. With more metallicity of $[\mathrm{M} / \mathrm{H}]=-4$, temperature in the density range $10^{5-8} \mathrm{~cm}^{-3}$ begins to deviate as a result of the enhanced $\mathrm{H}_{2}$ fraction due to its formation on dust grains. Finally, with metallicity of $[\mathrm{M} / \mathrm{H}]=-3$, this lower-density dip becomes deeper by the cooling by the fine-structure lines of $\mathrm{C}$ and $\mathrm{O}$. Although addition of further metallicity results in even deeper temperature dips, the overall nature of the thermal evolution remains the same. The evolutionary track of the temperature has two minima. One at lower density is by line-emission cooling, i.e., $\mathrm{H}_{2}$ and $\mathrm{HD}$ cooling at low $([\mathrm{M} / \mathrm{H}] \lesssim-3.5)$ metallicity, while $\mathrm{C}$, O fine-structure lines at higher metallicity. The other at higher density is by the dust cooling. Existence of the two temperature minima corresponds to two fragmentation epochs. Note that the fragmentation mass scales, which are given by the Jeans mass at the temperature minimum, depend on metallicity only moderately. The line-induced fragmentation is very high, $100-1000 M_{\odot}$, while the dust-induced fragmentation is low, $0.1-1 M_{\odot}$. Only the dust cooling enables low-mass fragmentation. Therefore, the dust is indispensable for low-mass star formation in a low-metallicity gas.

How much metallicity, or correctly speaking, amount of dust, is needed to cause fragmentation and thus produce low-mass cores? This threshold value is often called the critical metallicity in the literature (e.g., Schneider et al. 2002, Bromm \& Loeb 2003). To pin down this value, we have studied evolution of pre-stellar cores in the dust-cooling phase by SPH simulations (Tsuribe \& Omukai 2006; 2008). The results for $[\mathrm{M} / \mathrm{H}]=-4.5$ and -5.5 are shown in Figure 2. Here, for the dust model, we have assumed that produced in the supernovae of the first stars as in Schneider et al. (2006). In this model, the dust effects tend to be more emphasized than in the standard Galactic dust model used in Figure 1, owing to the smaller size (larger area for the same amount of mass) and more refractory composition. We assumed that the cores are initially moderately elongated and have some random velocity perturbations $(\mathrm{a}-1, \mathrm{~b}-1$ in Fig. 2). 

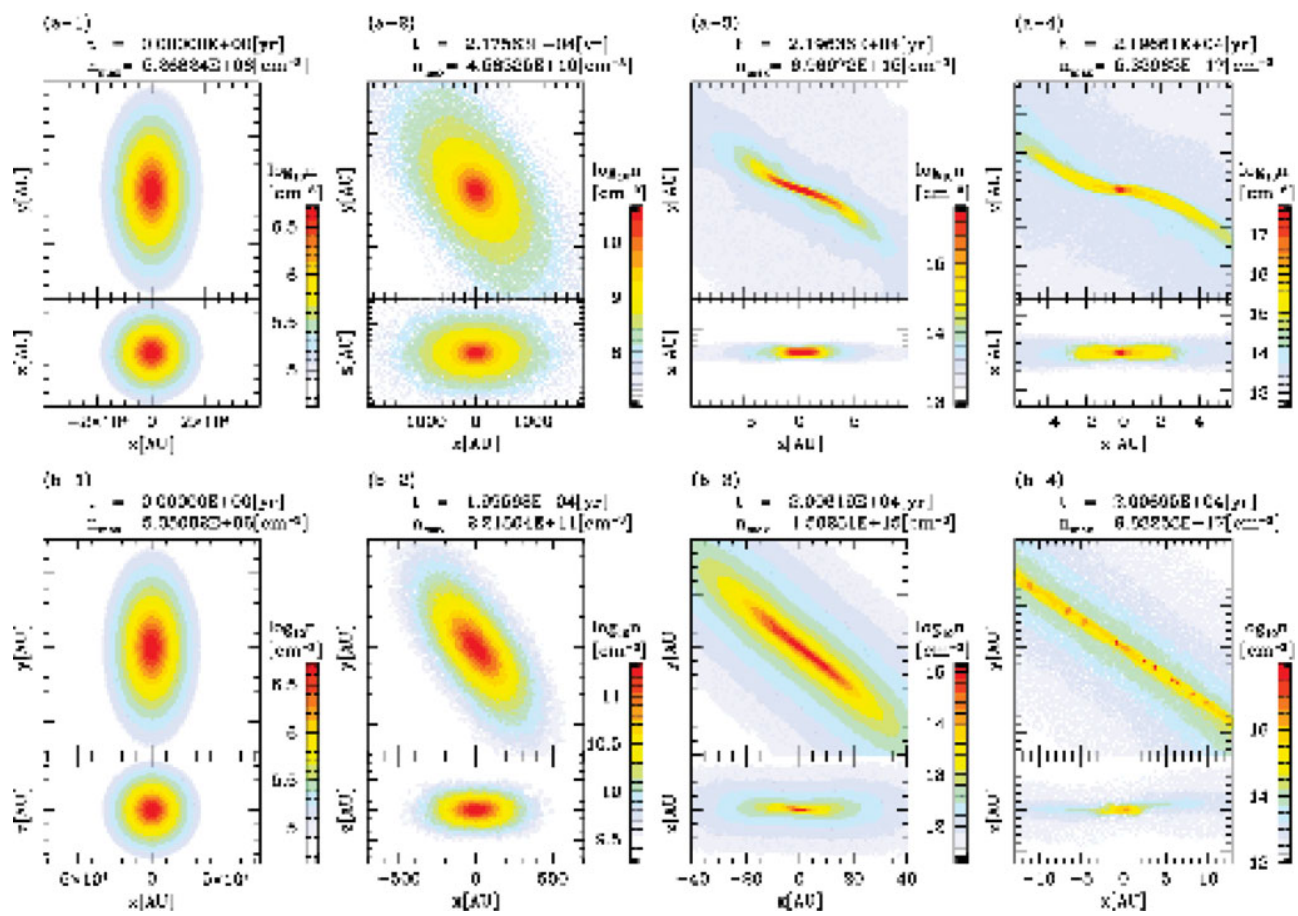

Figure 2. Evolution of cores with $(\mathrm{a})[\mathrm{M} / \mathrm{H}]=-4.5$ and $(\mathrm{b})-5.5$. The initial angular velocity is $0.1 \sqrt{4 \pi G \rho_{c}}$, where $\rho_{c}$ is the central density. Four different stages (panels 1-4) are shown from left to right. Density distributions in the $z=0$ plane (top), and the $y=0$ plane (bottom) are shown in each panel. The panels 1 show the initial states. The gray scale denotes the density in the logarithmic scale. The maximum number density and elapsed time are indicated on the top of each panel. (After Tsuribe \& Omukai 2008)

In the case of $[\mathrm{M} / \mathrm{H}]=-5.5$, which is shown in the bottom row (b-1..4), the core becomes more elongated (b-2,3) during the dust-cooling phase and finally fragments into many pieces (b-4). Similar fragmentation events are observed also at metallicity $[\mathrm{M} / \mathrm{H}]=$ -6 , but not at lower metallicity. Thus, for the first dust model, the critical metallicity is $[\mathrm{M} / \mathrm{H}]_{\mathrm{cr}} \simeq-6$. If we use the standard dust model, more dust is needed to cause fragmentation. Taking this into account, we conclude that the critical metallcity is in the range $[\mathrm{M} / \mathrm{H}]_{\mathrm{cr}}=-6$ to -5 . Only with slight metal enrichment, low-mass fragments can be produced as long as a sizable fraction of metals have condensed in the dust.

However, the cases between metallicity $[\mathrm{M} / \mathrm{H}]=-5$ and -4 are exceptions. As an example, the case of $[\mathrm{M} / \mathrm{H}]=-4.5$ is shown in the top row of Figure 2 (a-1..4). In this model, no fragmentation is observed in the dust-cooling phase, and thus only massive stars are expected to form. This absence of fragmentation is caused by a sudden temperature jump by the three-body $\mathrm{H}_{2}$ formation at $10^{8} \mathrm{~cm}^{-3}$, which can be seen also for $[\mathrm{M} / \mathrm{H}]=-4$ in Figure 1. This heating and resultant pressure increase makes the core spherical. Without the initial elongation, the core does not fragment subsequently.

\section{Accretion Evolution and Upper Limit on the Stellar Mass}

So far, we have seen the evolution during the pre-stellar collapse. Dense molecular cores are formed as a result of fragmentation, and inside of them, protostars are eventually 


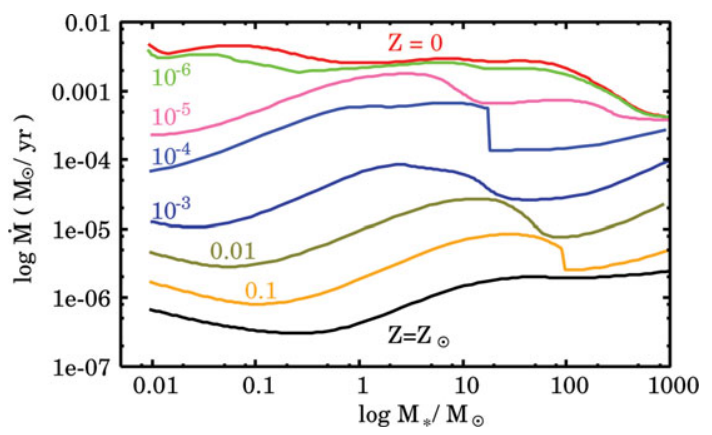

Figure 3. The fiducial protostellar accretion rates for different metallicities. (After Hosokawa \& Omukai 2009)

formed. Although the initial mass of the protostars is very small $\sim 10^{-2} M_{\odot}$, subsequently they grow in mass by accretion of the remnant of the dense core, or the envelope, and finally reach the main sequence stars.

One of the most important quantities in the accretion phase is the accretion rate, which is given by $\dot{M} \simeq c_{\mathrm{s}}^{3} / G \propto T^{3 / 2}$, where $c_{\mathrm{s}}$ is the sound speed in the pre-stellar cores. Using this relation, we can convert the temperature evolution of Figure 1 into the accretion rate as a function of the protostellar mass. We also assume that the cores are gravitationally unstable marginally, and then the instantaneous mass of the protostars $M_{*}$ is given by the Jeans mass in the cores. The "fiducial" accretion rate calculated in this way is shown in Figure 3. In low metallicity gas, the accretion rates are higher reflecting the higher temperature in pre-stellar cores. We calculated the protostellar evolution under the fiducial accretion rates by using the method of Palla \& Stahler (1991). In this method, the structure of a protostar is solved by solving ordinary stellar structure equations, while that of an accreting envelope is found by assuming the stationary flow. These two solutions are matched at the stellar surface by using the radiative shock condition. By increasing the protostellar mass in each time step, we can obtain an evolutionary sequence of the protostellar structure.

Figure 4 presents the evolution of the protostellar radius as a function of protostellar mass, which increases with time by accretion. The cases of metallicity $[\mathrm{M} / \mathrm{H}]=$ $-\infty,-6,-4,-2$, and 0 are shown from the top to bottom. The protostars have larger radii for lower metallicity, i.e., higher accretion rate. For higher accretion rate, the accreting envelope becomes denser, and the radiative cooling is less effective. Consequently, higher entropy gas is accreted and the star has a larger entropy and thus a larger radius. Despite this difference, the evolutionary features are rather similar in all cases, and can be characterized by four phases of (i) the adiabatic accretion, (ii) swelling, (iii) Kelvin-Helmholtz contraction, and (iv) main-sequence accretion phases. Initially, the Kelvin-Helmholtz $(\mathrm{KH})$ time is longer than the accretion time and the stars remain adiabatic. In this adiabatic accretion phase, the radius gradually increases. In the $Z_{\odot}$ case, the deuterium burning somewhat enhances the radial expansion in this phase. At some point, the luminosity increases, and then the KH time becomes comparable to the accretion time. The stars swell rapidly in the process of releasing the heat contained inside and subsequently begin the KH contraction. The central temperature continues to increse with the protostellar mass. Finally, the protostars begin the hydrogen burning and become the ordinary zero-age main-sequence stars. Note that, at lower metallicity, the protostars become more massive before the onset of hydrogen burning. 


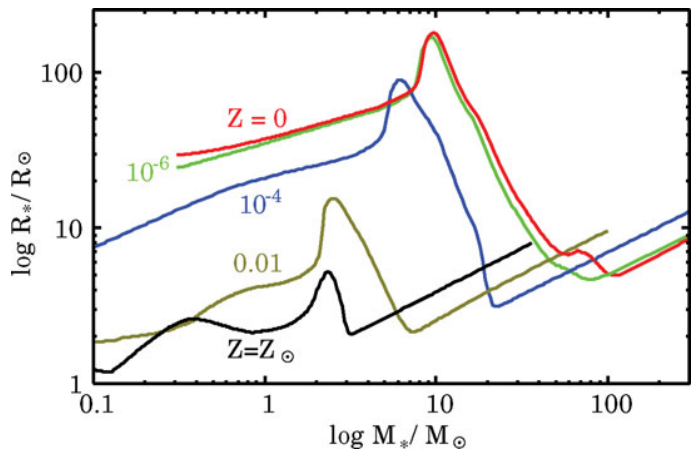

Figure 4. Evolution of the protostellar radii for different metallicities $Z=0,10^{-6}, 10^{-4}, 10^{-2}$ and $1 Z_{\odot}$. The fiducial accretion rates are adopted. (After Hosokawa \& Omukai 2009)

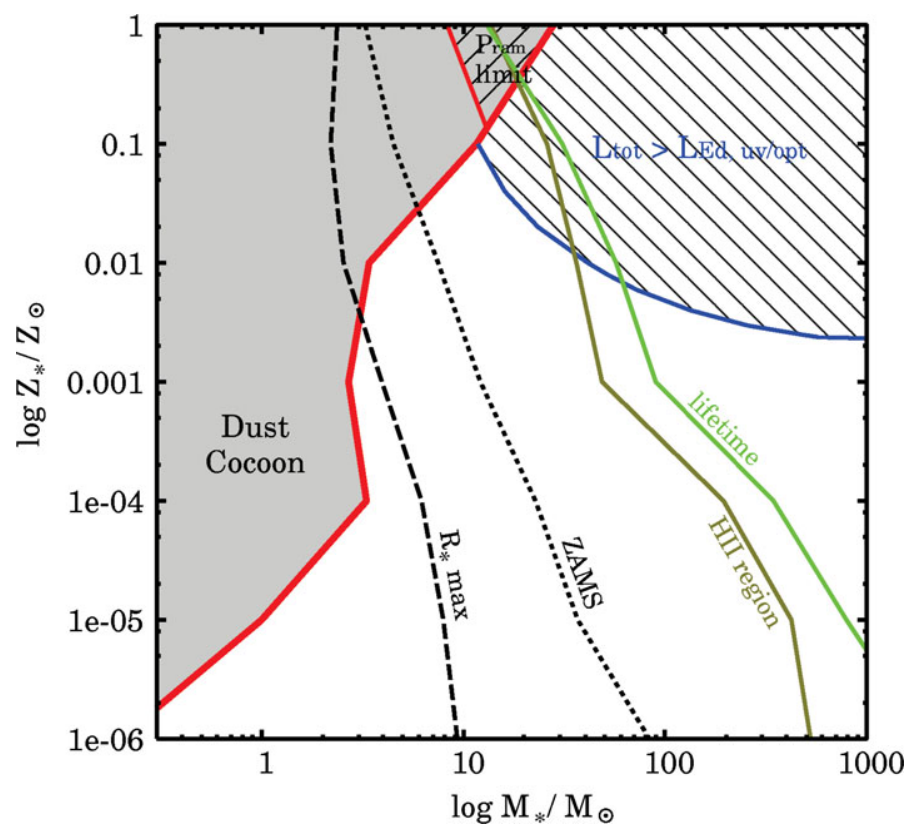

Figure 5. The upper mass limit of forming stars set by stellar feedback for different metallicities. The left gray region (with "dust cocoon") shows where the dust is optically thick. The right of it is optically thin region. The range where the accretion is halted by the radiation pressure onto the dusty envelope is indicated by the upper right hatched region ( $P_{\text {ram }}$ limit" for the optically

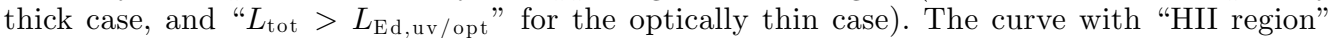
shows the limit by the expansion of the HII regions. The curve with "lifetime" (rightmost thin curve) is for the lifetime limit, where the MS lifetime exceeds the formation time. Also shown are the epochs of maximum protostellar radii in the swelling phase (dashed, with " $R_{*}$ max") and the MS arrival (dotted, with "ZAMS"). (After Hosokawa \& Omukai 2009)

Finally, we discuss the upper mass limit by the stellar feedback. Although the characteristic mass of dense cores is given by the fragmentation process, the cores are considred to be formed in a spectrum of mass. After the dust cooling has already caused the transition to the low-mass star formation mode, some fraction of massive cores can be 
formed. Even if the mass reservoir in the dense core is sufficient, however, the accretion cannot continue unlimitedly. When the central protostar becomes massive enough, its feedback onto the accreting envelope becomes very strong and stops the accretion eventually.

Here we consider the two kinds of stellar feedback processes to halt the accretion: the radiation pressure on the dusty envelope and the expansion of the HII regions. The stellar mass limit by these processes is shown in Figure 5. In low-metallicity gas, the higher accretion rate and lower opacity result in weaker feedback and then higher upper mass limit. This upper limit decreases with increasing metallicity gradually without remarkable sudden drops. For example, for metallicity above $0.01 Z_{\odot}$, the upper limit is set by radiation pressure, which is about 10 to $40 M_{\odot}$, assuming spherical accretion. For lower metallicity, the upper limit is set by the HII region expansion, which is as high as 40 to $500 M_{\odot}$.

\section{Summary}

We have studied the thermal evolution of low-metallicity gas and discussed its fragmentation properties. The line-emission cooling affects the thermal evolution only at low densities where the Jeans mass is still high ( $>10$ to $100 M_{\odot}$ ). The dust grains cause a sudden temperature drop at high density where $M_{\text {Jeans }} \lesssim 1 M_{\odot}$, which induces low-mass fragmentation. The critical metallicity for the dust-induced fragmentation is $[\mathrm{M} / \mathrm{H}]_{\mathrm{cr}} \sim-6$ to -5 . However, $\mathrm{H}_{2}$-formation heating prohibits low-mass fragmentation in the range $[\mathrm{M} / \mathrm{H}] \sim-5$ to -4 .

Also, we have studied the evolution of low-metallicity protostars and discussed the upper limit on the mass by stellar feedback. In low-metallicity gas, the high temperatures in star-forming clouds result in high accretion rates. Protostellar evolution is characterized by four evolutionary stages for all metallicities: 1 . the adiabatic accretion, 2 . swelling, 3 . $\mathrm{KH}$ contraction, and 4. MS accretion phases. Lower-metallicity protostars become more massive before the arrival to the main-sequence due to higher accretion. The upper limit on the stellar mass is 10 to $40 M_{\odot}$ set by radiation pressure feedback for $\mathrm{Z}>0.01 Z_{\odot}$, while it is 40 to $500 M_{\odot}$ set by expansion of HII regions for $\mathrm{Z}<0.01 Z_{\odot}$.

\section{Acknowledgements}

I thank the organizers for invitation to the beautiful town of Rapallo and a very enjoyable meeting.

\section{References}

Abel, T., Bryan, G. L., \& Norman, M. L. 2002, Science, 295, 93

Bromm, V., Coppi, P. S., \& Larson, R. B. 2002, ApJ, 564, 23

Bromm, V. \& Loeb, A. 2003, Nature, 425, 812

Hosokawa, T. \& Omukai, K., 2009, in preparation

Larson, R. B. 2005, MNRAS, 359, 211

McKee, C. \& Tan, J. 2008, ApJ, 681, 771

Omukai, K. \& Palla, F. 2003, ApJ, 589, 677

Omukai, K., Tsuribe, T., Schneider, R., \& Ferrara, A. 2005, ApJ, 626, 627

Palla, F. \& Stahler, S. W. 1991, ApJ, 375, 288 
Schneider, R., Ferrara, A., Natarajan, P., \& Omukai, K., 2002, ApJ, 571, 30

Schneider, R., Omukai, K., Inoue, A.-K., \& Ferrara, A. 2006, MNRAS, 369, 1437

Tsuribe, T. \& Omukai, K. 2006, ApJ, 642, L61

Tsuribe, T. \& Omukai, K. 2008, ApJ, 676, L45

Yoshida, N., Omukai, K., \& Hernquist, L. 2008, Science, 321, 669 